\title{
Carotid Artery Stenting via a Femoral Bypass Graft: Technical Note
}

Kentaro Hayashi M.D., Naoki Kitagawa M.D., Hideaki Takai M.D.*, Izumi Nagata M.D.

Department of Neurosurgery \& Cardiovascular Surgery*, Nagasaki University School of Medicine, Nagasaki, Japan

Correspondence to Kentaro Hayashi M.D.

Department of Neurosurgery, Nagasaki University School of Medicine

1-7-1 Sakamoto, Nagasaki-city 852-8501 Japan

Tel; +81-95-849-7375

Fax; +81-95-849-7378

e-mail; kenkuni@net.nagasaki-u.ac.jp 


\begin{abstract}
Background: We described a case of endoluminal stent placement for a cervical internal carotid artery stenosis in which access was obtained via the femoral bypass graft.
\end{abstract}

Case Description: A 70-year-old man with known disease of the carotid, peripheral, and coronary arteries as well as congestive heart failure presented for endoluminal revascularization of a severe right internal carotid artery stenosis. Transradial access was complicated by the left subclavian artery occlusion and hypercalcified aortic arch. Bilateral femoral artery was replaced with bypass graft because of atherosclerotic obstruction. An alternative approach was attempted via the exposed left femoral bypass graft.

Techniques: The left inguinal region was incised and the left common femoral-popliteal bypass graft was exposed. After placement of a purse string suture at the puncture site, the guiding sheath was introduced into the graft and positioned into the right common carotid artery. Stenting was successfully performed and the suture was tied after withdrawing the sheath.

Conclusions: This novel approach should be considered for endovascular procedures for which access to the carotid artery is limited.

Key words: carotid stenosis, carotid stent, bypass graft 


\section{Introduction}

Carotid artery stenting (CAS) has emerged as an acceptable alternative treatment for patient with occlusive carotid bifurcation disease. Recent advances in catheter technology have resulted in significantly improved access to carotid artery. However, transfemoral access to the carotid artery is occasionally limited by systemic atherosclerosis or previous surgical reconstruction (10). We present a technical report of a carotid artery stenting performed through aorto-femoral bypass graft. Search of the current literature has revealed no documentation of a previous carotid stenting performed by introducing catheter through synthetic prosthetic grafts.

\section{Case History}

\section{Presentation}

A 70-year-old man was admitted to our hospital for coronary artery bypass surgery. He had a history of bilateral bypass graft of femoral artery (abdominal aorto-left common femoral bypass, left common femoral-popliteal graft, right femoral patch-angioplasty, right common femoral-popliteal graft) ten years previously. Systemic evaluation of atherosclerosis demonstrated stenosis at the origin of the bilateral internal carotid artery and the right vertebral artery, and occlusion of the left subclavian artery (Fig. 1A). These lesions were accompanied with massive calcification (Fig. 1B). CT angiography of lower body demonstrated severe calcification of bilateral femoral artery and the 
bypass graft (Fig. 1C). Fig 1D showed schematic illustration of the femoral artery bypass. Hypoperfusion of the right cerebral hemisphere was revealed with single photon emission tomography. Diagnostic cerebral angiogram demonstrated severe stenosis at the origin of right carotid artery and moderate stenosis at the origin of left carotid artery (Fig. 2AB). Since extracorporeal circulation was required for the coronary bypass surgery and the intraoperative cerebral hypoperfusion was anticipated because of multiple cerebrovascular diseases, revascularization of the carotid stenosis was planed before cardiac surgery. Due to the increased risk of general anesthesia related to his severe comobidities and our limited experience with carotid endarterectomy under local anesthesia, stenting was considered. After discussion with vascular surgeon about the risks and benefits of the various options, including direct carotid artery approach, radial artery approach, and bypass graft approach, the bypass graft approach was employed.

\section{Procedural Technique}

Ticlopidine (300 mg) and aspirin (100 mg) were already administered orally on admission. Sedative and analgesic agents were administered intravenously. The left inguinal region was incised along vascular pulsation approximately $4 \mathrm{~cm}$ and the left common femoral-popliteal bypass graft (Bard Sauvage Bionit EXS Velour Vascular Prosthesis 6 mm, C.R.Bard Inc, Billerica, MA) was exposed (Fig. 3A). The bypass graft was supported with ring structure to avoid vessel collapse or kink. The surface part of two rings were removed to secure puncture area (Fig. 3B). Then, purse string suture was put on the graft at the puncture site with 5-0 proline (Fig. 3C). The graft was punctured 
with18-G needle and a guide wire was introduced through the needle (Fig. 3D). The guide wire was carefully advanced into the abdominal aorta under fluoroscopic control. Then, the needle was withdrawn along the wire and the 6-F 90-cm guiding sheath (Shuttle; Cook, Inc. Bloomington, IN) with inner dilator was gently threaded over the guide wire into the graft. The guiding sheath was positioned using coaxial system into the right common carotid artery and the purse string suture was tensed to avoid bleeding from puncture site (Fig. 3E). After establishment of arterial access, heparin was administered to maintain an activated coagulation time of approximately 300 seconds during the procedure. A self-expandable Wallstent (Boston Scientific Co., Natick, MA) was placed under cerebral protection using PercuSurge Guardwire plus (PercuSurge, Minneapolis, MN). The suture was tied after withdrawing the sheath (Fig. 3F). Finally, the wound was closed (Fig. 3G). Intraprocedural bleeding was well controlled despite dual-antiplatelet agents. Postprocedural angiography showed satisfactory widening of the stenosis (Fig. 3H).

\section{Postprocedural Course}

The patient stayed on bed rest for six hours with the punctured extremity extended. Postprocedural course was uneventful and cardiac surgery was subsequently performed. The ultrasound echo examination one month later showed patency of the punctured bypass graft.

\section{Discussion}


CAS is an alternative revascularization technique for extracranial carotid stenotic disease. Since stenting is less invasive comparing with endarterectomy, advanced age patients or patient who have serious complication are more likely to be candidate for CAS. Since the number of patients who have undergone reconstructive vascular surgery is continuously increasing, we are faced with an increasing demand for the carotid revascularization of patient with aorto-femoral bypass graft.

For those patients, traditional approach has been through the radial route or direct carotid approach to avoid direct puncture of the graft site because of possible postprocedural complication. Direct carotid puncture is an important option even in CAS when a guiding catheter is not advanced into common carotid artery because of arterial tortuosity (9). However, the direct carotid puncture requires greater skill and is more cumbersome. Hemostasis after the procedure is not easy and hematoma formation from the puncture site may cause serious complications (2). Recent reports have suggested the safety and feasibility of transradial approach for coronary stenting as well as CAS $(3,8)$. However, this approach requires special effort in the optimization of the backup and the stabilization of the guiding catheter. This procedure often requires a good strategy, appropriate catheter manipulations, and the use of specific technical maneuvers such as buddy wire. Moreover, nerve injury or postoperative radial artery occlusion remains a major concern. In this patient, transradial approach was not suitable because of the left subclavian artery obstruction and the hypercalcified brachiocephalic trunk (Fig. 1AB). In this report, the authors have employed CAS via the left 
aorto-femoral graft.

Fear of direct graft puncture has been based on the following potential hazards: uncontrollable bleeding and hematoma formation because of the non-vascular nature of the punctured graft, disruption to the anastomotic suture line and consequent false aneurysm formation, infection of the graft site necessitating surgical intervention and disruption of the pseudo-intima with possible thrombogenesis $(6,11)$. Gallagher et al reported the safety of percutaneous transfemoral coronary and peripheral procedures via aorto-femoral synthetic vascular grafts (7). Adverse events related to vascular access occurred in 7 of 123 procedures (5.7\%), including blood transfusion (4.1\%), thrombotic occlusion (1.6\%), transient limb ischemia (0.8\%), and retroperitoneal hemorrhage (0.8\%). No deaths, graft infections, or pseudoaneurysms were reported. Although the aorto-femoral vascular access complication rate was higher, it remains an acceptable approach $(1,4)$.

We would like to emphasize the superiority of the cut-down procedure over percutaneous access on bleeding from puncture site and lower extremity ischemia. Since larger devices are required for CAS comparing with coronary intervention, the damage of the synthetic prosthetic graft would be larger. In the clinical protocol, antiplatelet therapy is needed before and after CAS to prevent acute or delayed stent thrombosis. Furthermore, heparin is administered to maintain an activated coagulation time of approximately 300 seconds during the procedure. As a result, the most common complication is bleeding from the access site. To avoid local hemorrhage or retroperitoneal hemorrhage resulting to blood transfusion, the bypass was exposed and 
purse string suture was placed. Then, homeostasis was completed by tying the suture. Intraprocedural bleeding was not significant despite pretreatment with dual-antiplatelet agents. Another significant complication is catheterization-related graft thrombosis (5, 7). This may be related to the compression of puncture site to prevent bleeding. In this case, postprocedural compression to the access site was not required because of cut-down procedure. Femoral doppler ultrasonography obtained one month after the procedure demonstrated excellent patency of the bypass graft.

\section{Conclusion}

A femoral bypass graft approach with a small incision was performed safely, which suggests that intervention through bypass graft for CAS may be feasible when heavy systemic calcifications hinder more traditional approaches.

\section{References}

1. AbuRahma AF, Robinson PA, Boland JP: Safety of arteriography by direct puncture of a vascular prosthesis. Am J Surg 1992;164:233-6.

2. Blanc R, Mounayer C, Piotin M, Sadik JC, Spelle L, Moret J: Hemostatic closure device after carotid puncture for stent and coil placement in an intracranial aneurysm: technical note. AJNR Am J Neuroradiol 2002;23:978-81. 
3. Burzotta F, Hamon M, Trani C, Kiemeneij F: Direct coronary stenting by transradial approach: rationale and technical issues. Catheter Cardiovasc Interv 2004;63:215-9.

4. Cowling MG, Belli AM, Buckenham TM: Evaluation and complications of direct graft puncture in thrombolysis and other interventional techniques. Cardiovasc Intervent Radiol 1996;19:82-4.

5. Da Silva JR, Eckstein MR, Kelemouridis V, Waltman AC, Brewster DC, Abbott WM, Athanasoulis CA: Aortofemoral bypass grafts: safety of percutaneous puncture. J Vasc Surg 1984;1:642-65.

6. Eisenberg RL, Mani RL, McDonald EJ: The complication rate of catheter angiography by direct puncture through aorto-femoral bypass grafts. AJR Am J Roentgenol 1976;126:814-6.

7. Gallagher MJ, Dixon SR, Safian RD, Madala M, Abraham R, Rimar SD, Mattichak SJ, O'Neill WW, Kahn JK: Safety of percutaneous transfemoral coronary and peripheral procedures via aortofemoral synthetic vascular grafts. Am J Cardiol 2005;96:382-5.

8. Levy EI, Kim SH, Bendok BR, Qureshi AI, Guterman LR, Hopkins LN: Transradial stenting of the cervical internal carotid artery: technical case report. Neurosurgery 
2003;53:448-51.

9. Perez-Arjona EA, DelProsto Z, Fessler RD: Direct percutaneous carotid artery stenting with distal protection: technical case report. Neurol Res 2004;26:338-41.

10. Wang H, Swischuk JL, Fraser K, Alvernia J, Lanzino G: Transaxillary carotid stenting: technical case report. Neurosurgery 2005;56(2 Suppl):E441.

11. Zajko AB, McLean GK, Freiman DB, Oleaga JA, Ring EJ: Percutaneous puncture of venous bypass grafts for transluminal angioplasty. AJR Am J Roentgenol 1981;137:799-802. 


\section{Figure legends}

Fig. 1

MR angiography of upper body revealed stenotic lesion at the bilateral carotid arteries and right vertebral artery as well as occlusion of the left subclavian artery (A). CT angiography showed severe calcification at the lesion (B). CT angiography of lower body (C) demonstrated severe calcification of bilateral femoral artery and bypass graft (arrow). Schematic illustration (D) of femoral artery bypass (abdominal aorto-left common femoral bypass, left common femoral-popliteal graft, right femoral patch-angioplasty, right common femoral-popliteal graft). The Black parts indicate atherosclerotic occlusion or stenosis. The border parts indicate bypass graft.

Fig. 2

Lateral right carotid angiogram demonstrated high grade stenosis at the origin of the internal carotid artery (A). Lateral left carotid angiogram showed moderate stenosis at the origin of the internal carotid artery (B).

Fig. 3

After the left common femoral-popliteal bypass graft was carefully exposed, surface part of the ring was removed $(\mathrm{A}, \mathrm{B})$. Then, purse string suture was put on the graft at the puncture site with 5-0 proline (C). The graft was directly punctured with a needle (D). A 6-French Shuttle sheath was carefully advanced into the right common carotid artery (E). 
After withdrawing the sheath, the proline was sutured quickly (F). The wound was closed (G). Postprocedural angiogram showed satisfactory widening of the stenosis $(H)$. 
Fig. 1A

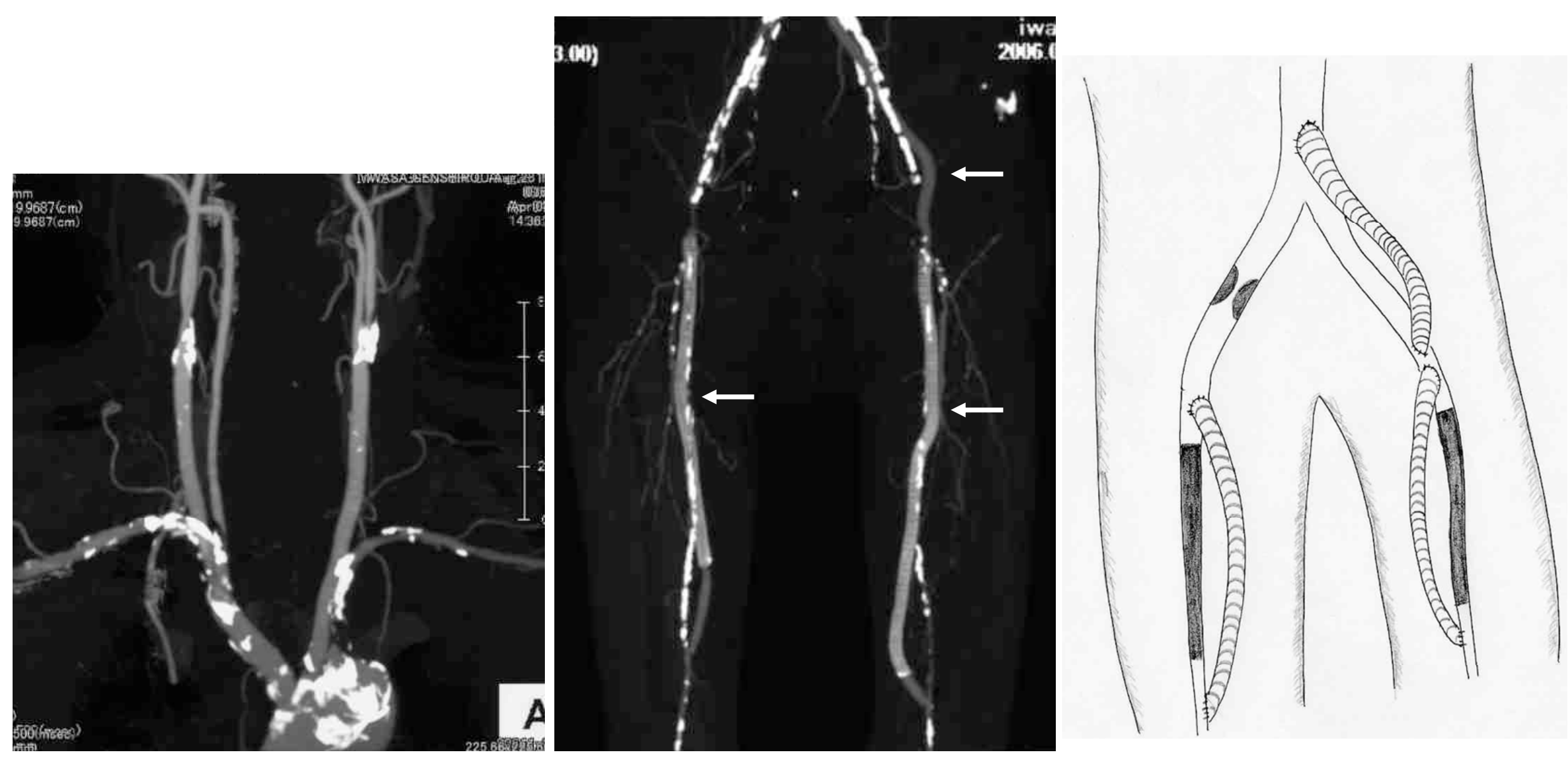

Fig. 1B

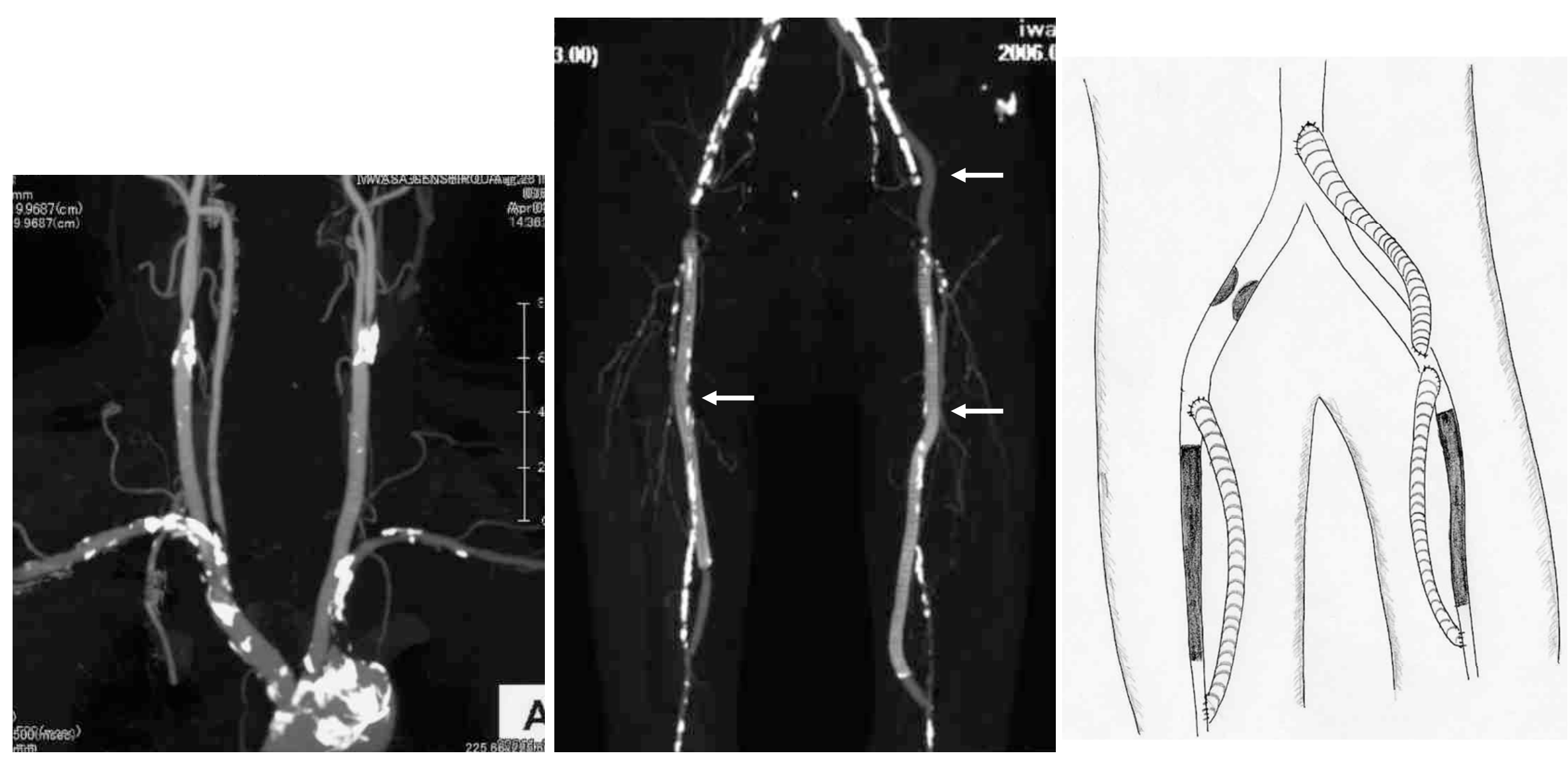

Fig. 1C 
Fig. 2A

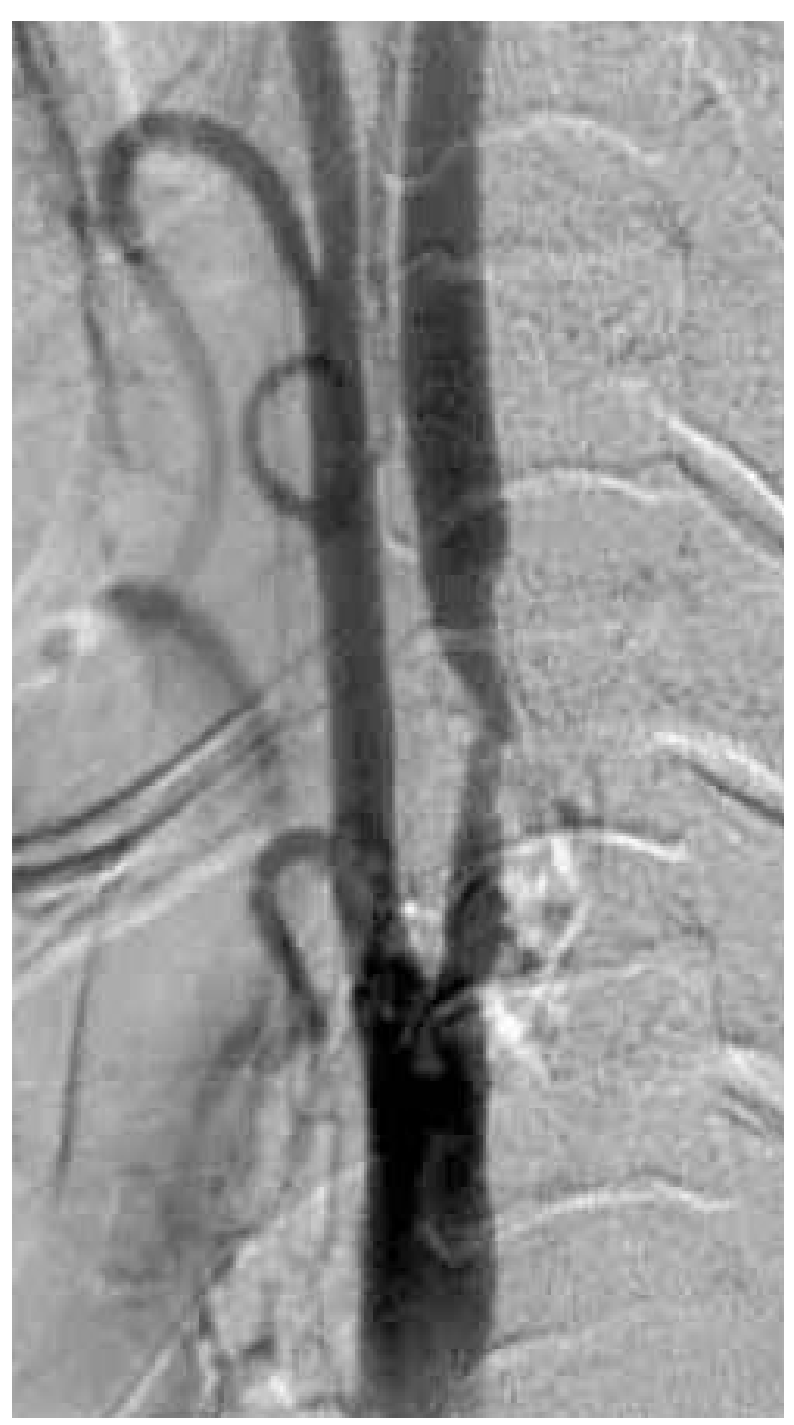

Fig. 2B

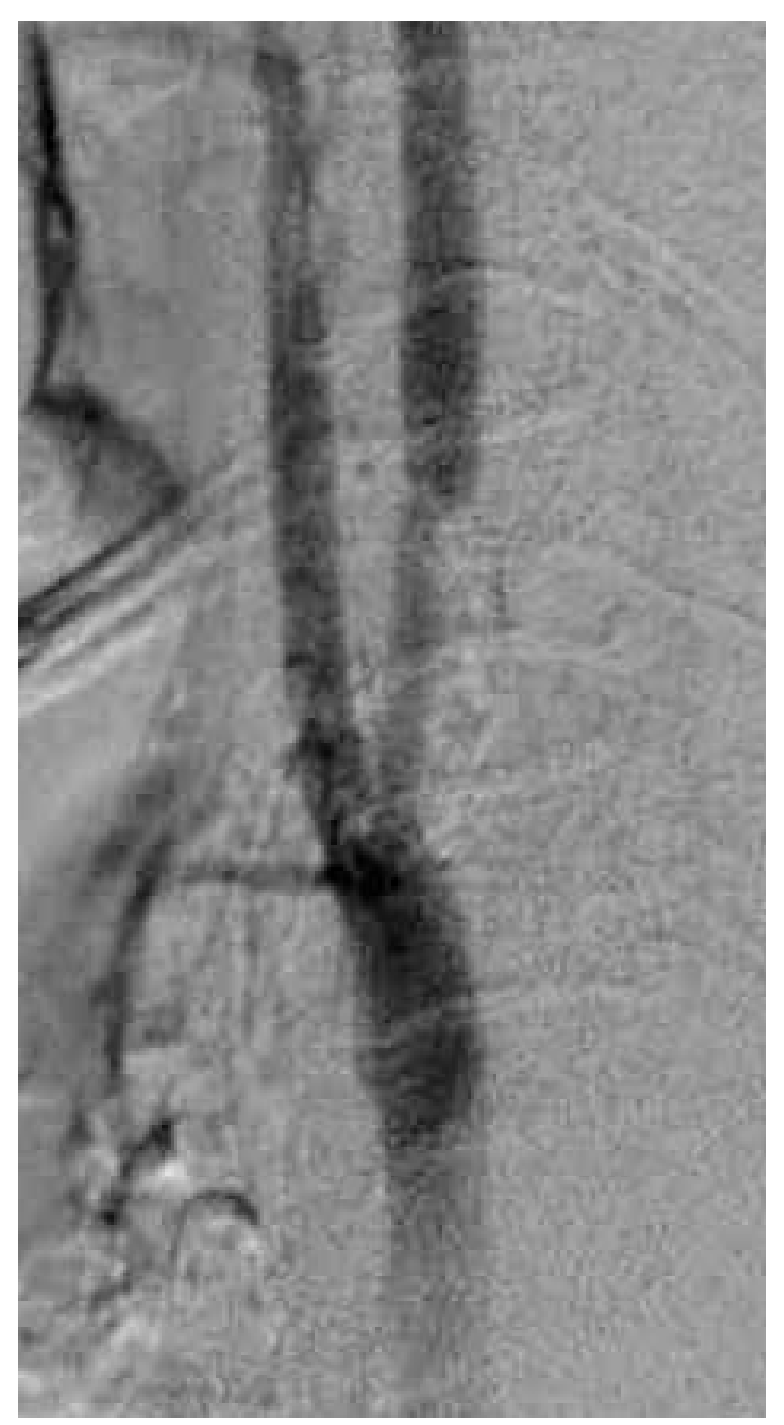


Fig. 3A

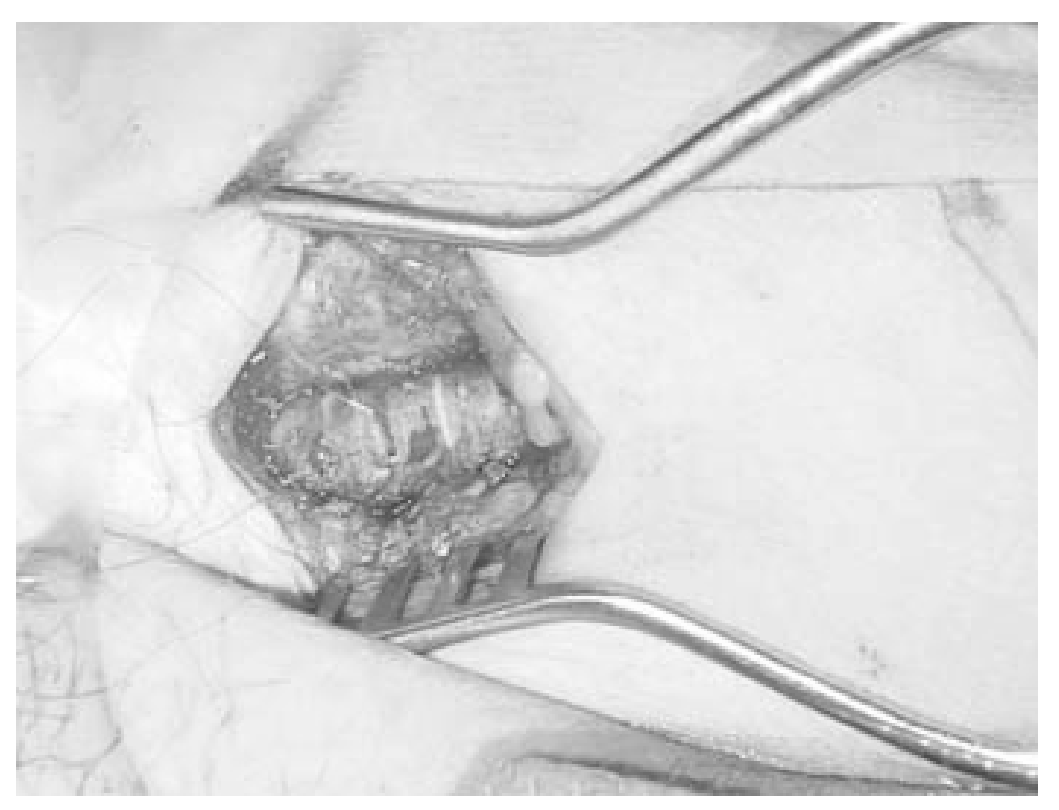

Fig. 3D

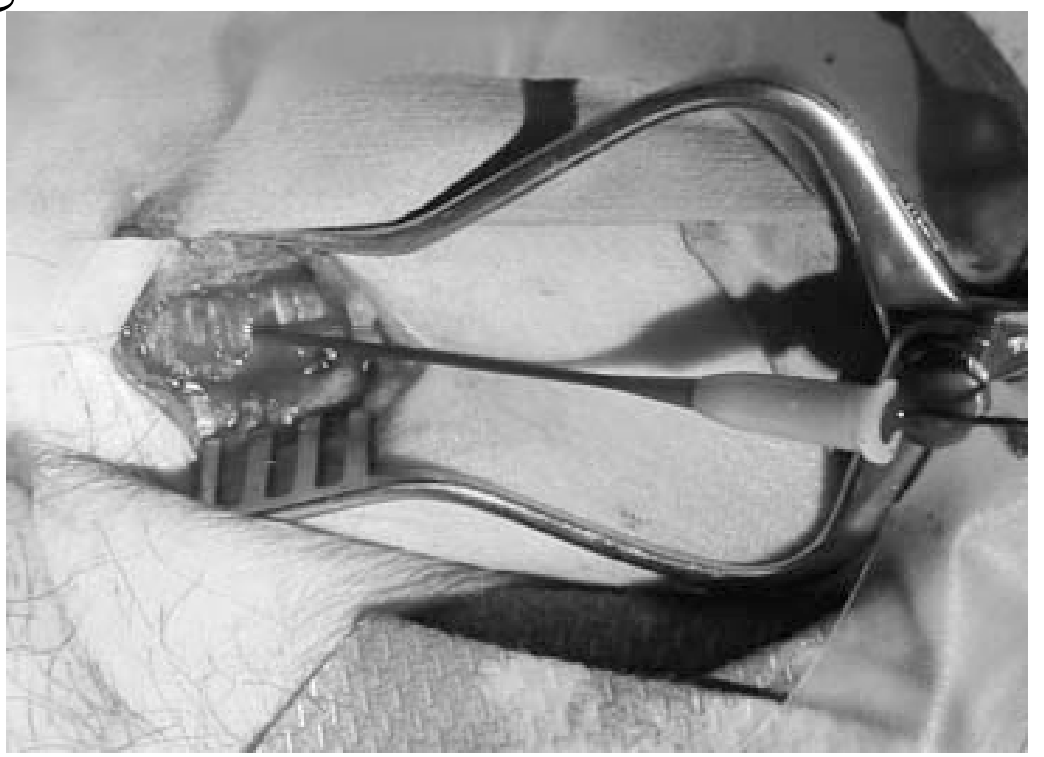

Fig. 3B

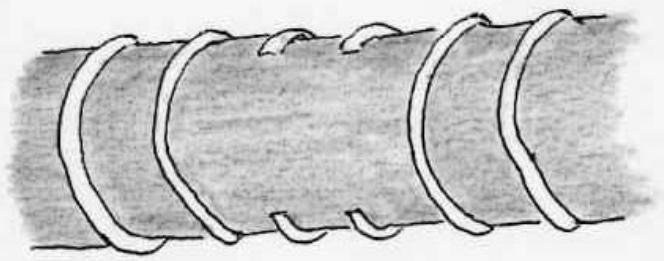

Fig. 3C

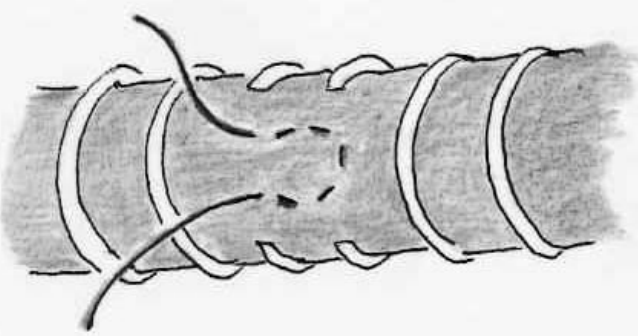

Fig. 3E

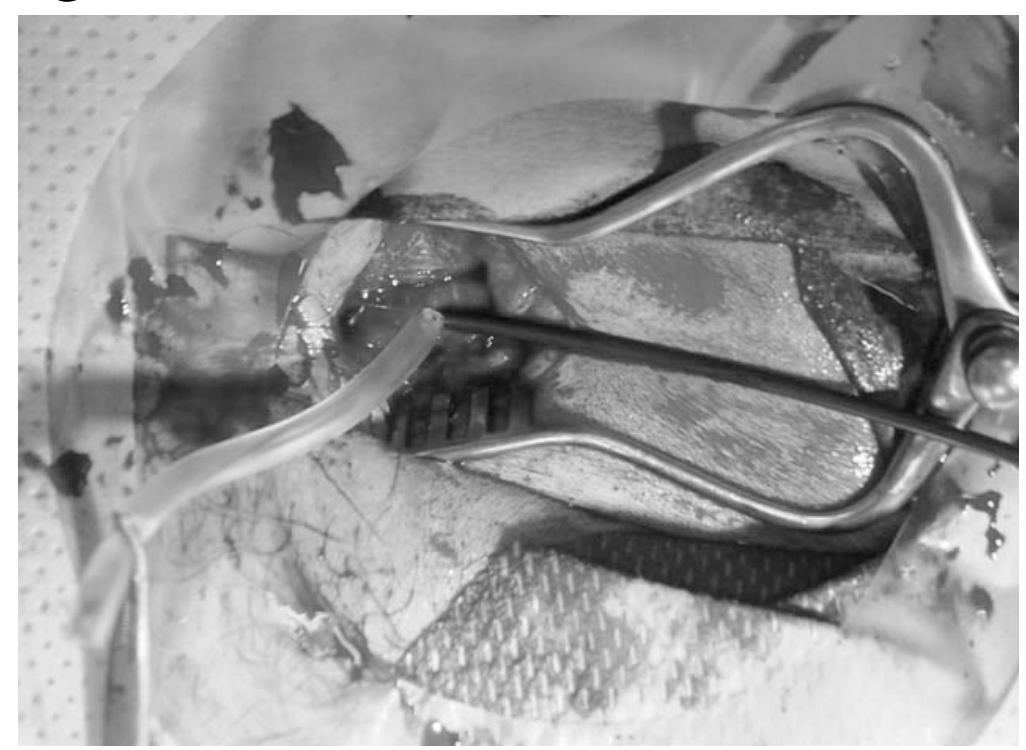


Fig. 3F

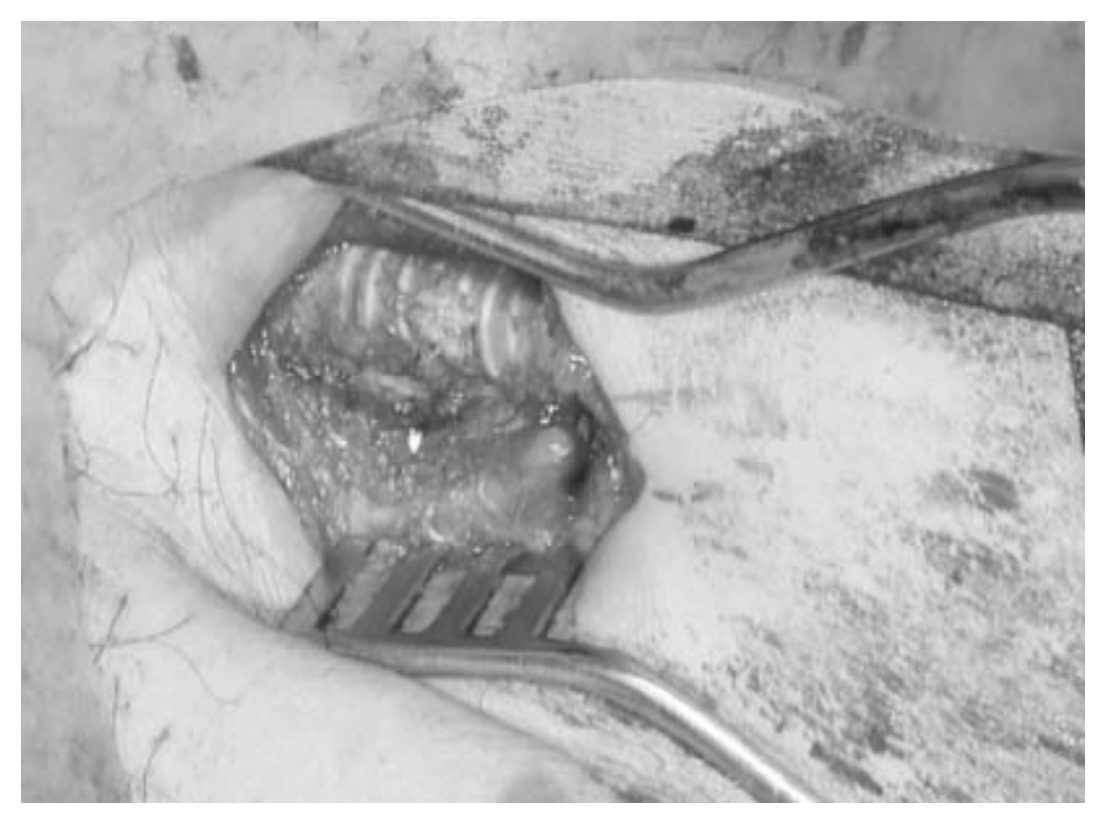

Fig. 3G

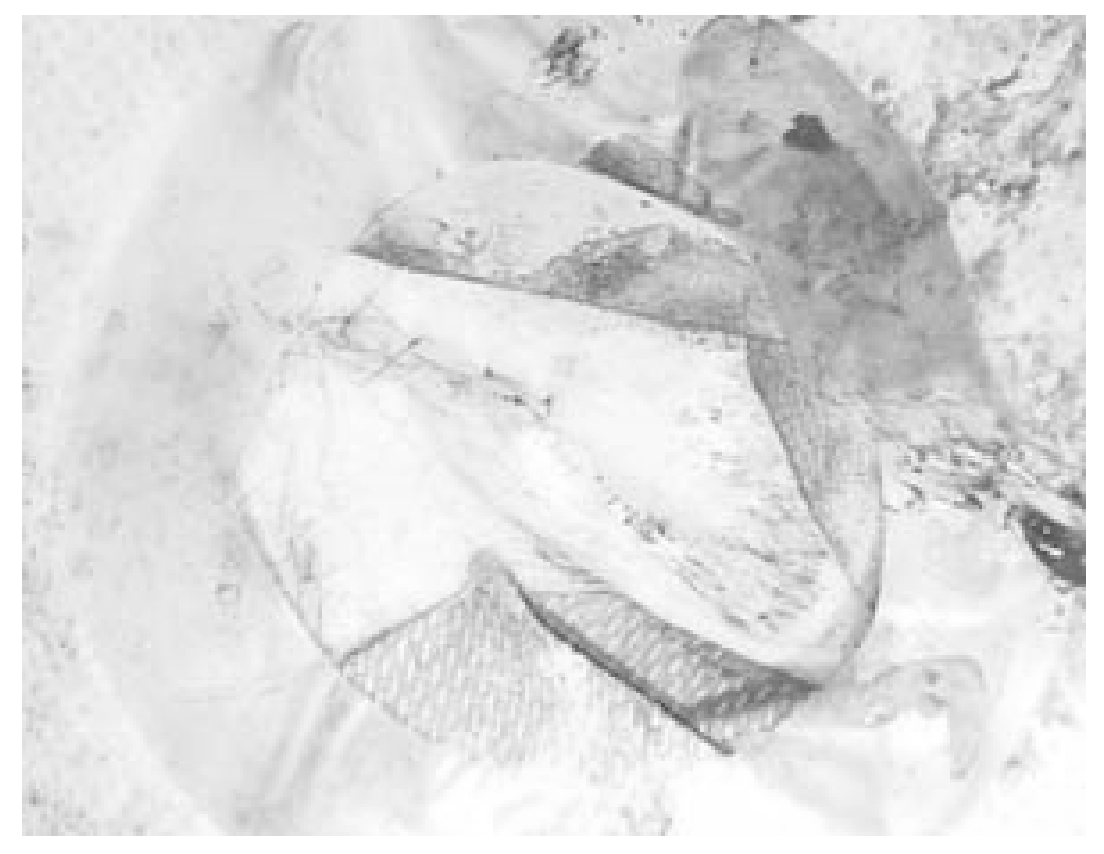

Fig. $3 \mathrm{H}$

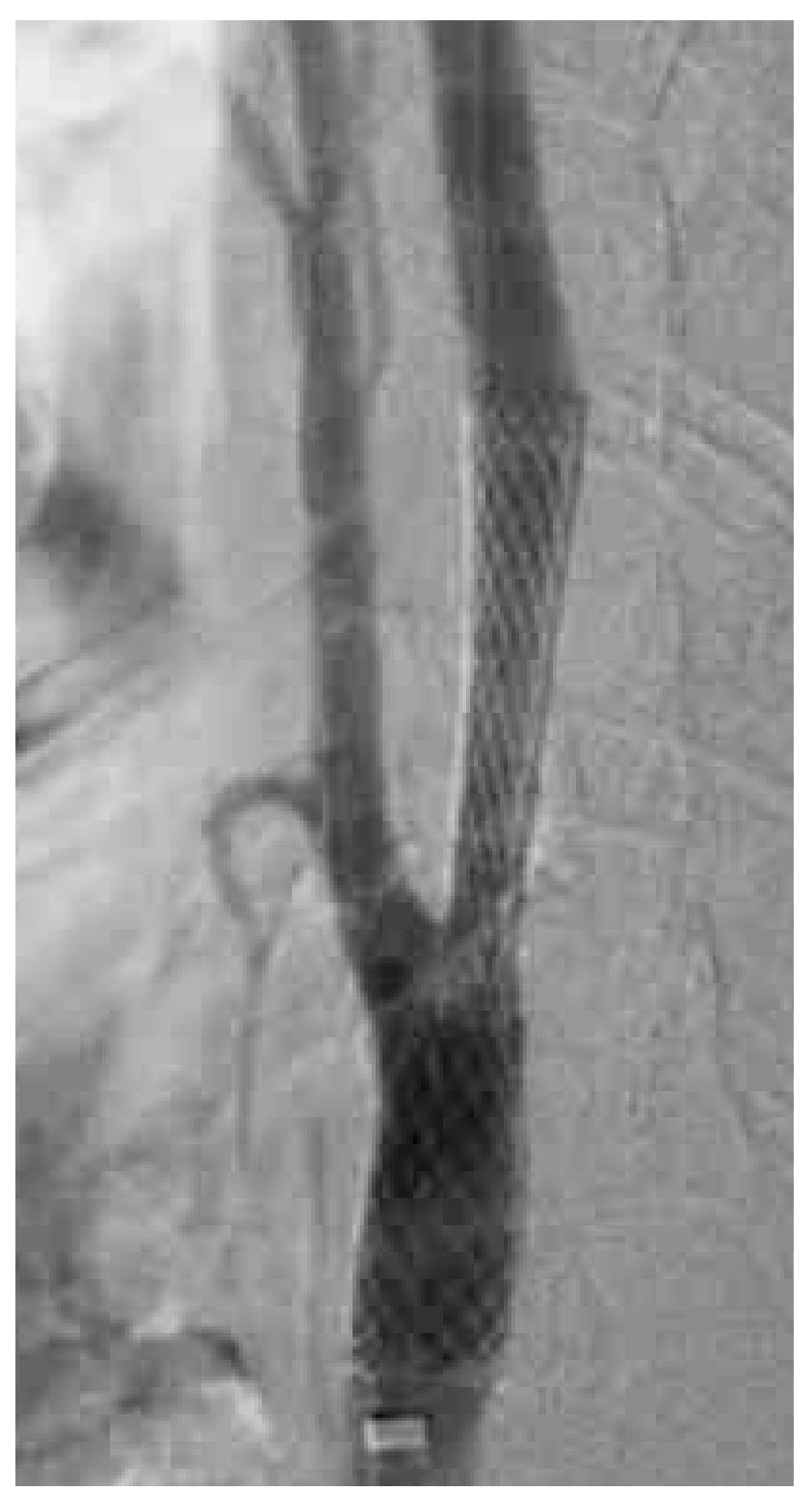

